\title{
Étude sur le manuscrit 1 de la Bibliothèque municipale de Semur-en-Auxois : enluminure de manuscrit et réforme monastique.
}

\section{Daniel Russo}

\section{(2) OpenEdition \\ 1 Journals \\ Édition électronique \\ URL : https://journals.openedition.org/cem/368 \\ DOI : $10.4000 /$ cem.368 \\ ISSN : 1954-3093 \\ Éditeur \\ Centre d'études médiévales Saint-Germain d'Auxerre}

\section{Édition imprimée}

Date de publication : 15 août 2006

ISSN : 1623-5770

Référence électronique

Daniel Russo, «Étude sur le manuscrit 1 de la Bibliothèque municipale de Semur-en-Auxois :

enluminure de manuscrit et réforme monastique. ", Bulletin du centre d'études médiévales d'Auxerre I BUCEMA [En ligne], 10 | 2006, mis en ligne le 13 octobre 2006, consulté le 22 septembre 2022. URL http://journals.openedition.org/cem/368 ; DOI : https://doi.org/10.4000/cem.368

Ce document a été généré automatiquement le 22 septembre 2022.

\section{(c) (i) (2) (2)}

Creative Commons - Attribution - Pas d'Utilisation Commerciale - Partage dans les Mêmes Conditions 4.0 International - CC BY-NC-SA 4.0

https://creativecommons.org/licenses/by-nc-sa/4.0/ 


\title{
Étude sur le manuscrit 1 de la Bibliothèque municipale de Semur- en-Auxois : enluminure de manuscrit et réforme monastique.
}

\author{
Daniel Russo
}

1 Les anciennes abbayes de Moutiers-Saint-Jean (Saint-Jean-de-Réome), de Flavigny et de Sainte-Reine ont contribué largement à nourrir le fond de la Bibliothèque municipale de Semur-en-Auxois ${ }^{1}$. C'est notamment le cas du très beau manuscrit 1 , le plus ancien, contenant la Vie de saint Jean de Réome et des Mélanges sur l'histoire de Moutiers-Saint-Jean. Longtemps daté du début $\mathrm{du} \mathrm{x}^{\mathrm{e}}$ siècle, il a été replacé par François Avril dans la chronologie de l'an mil, autour du début du $\mathrm{xI}^{\mathrm{e}}$ siècle. Dans l'écriture, des changements sont observés à partir du feuillet 52 verso où l'on passe de la caroline pure à des caractères dont certains aspects, la verticalité et la forme générale, sont à rapprocher de ceux d'un copiste actif, à la même époque, dans le scriptorium du Mont-Saint-Michel. Dans la mesure où l'abbaye de Fécamp, très liée au Mont, était alors réformée par l'abbé de Saint-Bénigne de Dijon, Guillaume de Volpiano (962-1031), il paraîtrait vraisemblable d'établir une relation plus précise entre le scribe du manuscrit de Semur, peut-être un moine bourguignon, et le scribe montois. Suivant cette hypothèse de datation, fondée sur des arguments paléographiques et stylistiques, Guy Lobrichon a situé le manuscrit 1 de Semur dans l'environnement plus large des grandes commandes de prestige passées, au cours de la première décennie $\mathrm{du} \mathrm{xI}^{\mathrm{e}}$ siècle, par les abbés réformateurs dans la mouvance de Guillaume : par exemple, l'abbé Heldric d'Auxerre et le foyer auxerrois ${ }^{2}$.

2 Le manuscrit fut décoré de belles initiales et de lettrines zoomorphes. Du feuillet 45 verso au feuillet 49 recto., il présente une des premières notations musicales sous forme de neumes, qui évoquent l'ornementation d'une mélodie de plain-chant.

3 En retenant la datation autour de l'an mil, sans doute au tout début du $\mathrm{XI}^{\mathrm{e}}$ siècle, nous voudrions insister ici sur l'importance de la lettre illustrée dans l'ambiance monastique 
réformée. Nos remarques s'inscrivent dans une enquête qui porte sur les $\mathrm{XI}^{\mathrm{e}}-\mathrm{XII}^{\mathrm{e}}$ siècles et des manuscrits issus des mêmes courants réformateurs en Bourgogne ${ }^{3}$. Pour le manuscrit 1 de Semur comme pour les autres exemples en cours d'étude, nous voudrions montrer comment tous ces objets précieux valent par eux-mêmes, à travers les formes et les décors choisis, et pas seulement en rapport avec un sens imposé de l'extérieur ${ }^{4}$. Nous suivrons ce projet selon deux approches privilégiées.

La vie exemplaire du moine. Formes et lettres

4 Topos hagiographique et mode de composition figurée vont de pair pour exalter le parcours de Jean de Réome à la suite de Jésus-Christ, fondateur de la petite communauté monastique, et pour construire la fama sanctitatis du personnage à vénérer. D’après son biographe, Jonas de Bobbio, dans sa Vita Joannis recueillie dans le manuscrit, c'est dans le diocèse de Langres que le jeune Jean décida, alors âgé d'une vingtaine d'années, de se retirer au cœur de la grande forêt qui s'étendait entre l'Armançon et le Serein, dans les ruines d'une habitation appelée Réome, près de Courtangy, d'où il était originaire. C'était vers le milieu du v viècle, et Jean venait d'une famille, celle des Hilarii, bien établie dans le Dijonnais et l'Auxois. Dans sa retraite le rejoignit un groupe d'ascètes qui poursuivait les mêmes buts que lui : la solitude pour mieux se mettre en quête de Dieu. Le groupe grossit; Jean se lassa et partit avec deux moines en direction de Lérins ${ }^{5}$, l'île des saints d'où, découvert par un visiteur trop importun, il finit par revenir au bout d'une année, rappelé par l'évêque Grégoire. Il mourut dans son monastère vers 540 , âgé de cent vingt ans. Le texte reproduit en préambule à la Vie a été reporté au feuillet 1 verso dans un encadrement en bandeaux de couleur bleue et mauve, en alternance, construit en rectangle bien tracé. Les caractères, en latin, Incipit ecce patris gestorum vita Johannis..., imitent la capitale latine antique et se trouvent circonscrits à l'intérieur de ce format régulier organisé en bandes parallèles. Le procédé de composition est tout à fait analogue à celui de l'exposition littéraire en forme de tableau reconnu et authentifié. Dans un style qui reste proche de celui des feuillets incipit à grands feuillages et bandes superposées de l'époque des manuscrits insulaires, le but poursuivi est de veiller à ennoblir le mot retranscrit en le situant à la fois matériellement ${ }^{6}$ et analogiquement dans la création universelle. Le scribe a le souci, majeur, d'illustrer le geste de l'homme dans la transcription du verbe de Dieu. Ici, sur le feuillet 1 verso, sa tâche consiste à rendre forme à ce qu'il considère comme de l'informe en recourant à des usages qui s'apparentent de très près aux modes (modi) et aux savoirs des grammairiens, reconnus et admis depuis longtemps ${ }^{7}$. Parfois aussi, sur le feuillet 15 verso par exemple, les bandeaux de couleur sont insérés au cadre en une suite de motifs entrelacés à anneaux, en trame à chaînons, ou en une suite d'anneaux attachés. De part et d'autre, les montants forment les deux hampes de l'initiale « $\mathrm{I}$ » et s'achèvent par des entrelacs tandis qu'au milieu du feuillet, sur le fond de parchemin, l'inscription est reportée en capitale latine : Incipiunt lectiones in nativitate sancti Johannis, confessoris... Lectio prima. Le tracé des lettres se ressent des influences dues à l'évolution des alphabets à lettres arrondies, d'abord l'écriture onciale ( $v^{e}$ siècle), puis l'écriture caroline ( $x^{e}$ siècle), correspondant à deux moments majeurs dans l'histoire de l'abbaye.

5 D'autres dispositifs agissent de même, précisent et dévoilent les contenus, provoquent librement d'autres associations, parfois inédites, presque toujours décontextualisées, puisqu'il serait vain de prétendre ne pas voir que le processus en œuvre est un processus d'engendrement formel qui finit par se reproduire lui-même, un peu à la 
manière du commentaire sur la lettre, infini et toujours recommencé ${ }^{8}$. Les caractères écrits peuvent être distribués en bandeaux comme de petites pancartes à fond coloré, bleu et mauve, sur le feuillet 2 recto, ou en colonnes, sur le feuillet 16 recto, par exemple (fig.). Dans les deux cas une grande initiale « $\mathrm{V}$ ", à monstres serpentins et à feuilles d'acanthe, sert d'ancrage au texte écrit. Formes et figures reviennent au répertoire pratiqué à l'époque du décor insulaire ( $\mathrm{VII}^{\mathrm{e}}-\mathrm{VIII}{ }^{\mathrm{e}}$ siècles), repris et adapté avec soin à l'époque carolingienne. Sur le feuillet 2 recto, ce sont des aigles et des lions ; sur le feuillet 16 recto, en haut, ce sont peut-être des renards, un monstre à cou de serpent, qui s'enroulent à la branche $\mathrm{du}$ " $\mathrm{V} »$, alors que de l'autre côté un aigle semble poursuivre un caprin. Dans les deux cas aussi, la base de la composition est formée d'une large feuille d'acanthe. Il faudrait analyser jusqu'aux moindres détails pour saisir l'entrecroisement des lignes et le chatoiement des couleurs appliquées en taches successives et pleines sur telle ou telle partie de lettre. Qu'il nous suffise de remarquer, à ce point de l'enquête, que la figure n'est pas un élément isolé du reste, mais n'agit que dans la série de toutes les autres, petites ou grandes, et qu'ensemble avec les autres elle montre, c'est-à-dire qu'elle fait voir, le prestige du texte transcrit. De plus, elle n'est jamais l'illustration, au sens trivial du terme, du contenu d'un texte : elle est un autre discours qui entre en consonance avec les autres figures ou formes disséminées dans les feuillets du manuscrit. Il arrive souvent que la lettre figurée prenne le dessus sur le texte mis par écrit, toujours pour construire la légende hagiographique de saint Jean de Réome.

L'initiative de l'initiale

6 Plus que de sens importé, voire imposé, de l'extérieur, il vaudrait mieux parler d'imagination "littérale » ou de la part inventive du scribe qui, à l'époque, est aussi l'enlumineur. Il est vrai que cette tradition formelle est inscrite dans une longue tradition théologique dont le modèle, sans doute le plus abouti, nous est fourni par les Étymologies d'Isidore de Séville (570-636), au début du viI ${ }^{\mathrm{e}}$ siècle. Sa conception de la graphie reposait sur deux principes, que nous retrouvons bien à l'œuvre dans la composition des lettres figurées à l'intérieur du manuscrit de Semur-en-Auxois: le respect de la parole divine à retranscrire comme une unité visuelle de signes référant au mystère, qu'il s'agit dès lors de pointer plutôt que de dire ; les caractères écrits lus comme les supports matériels de la Révélation. Nous n'insisterons plus sur ce que cette conception double, à bien des égards ressentie aujourd'hui comme antithétique, entraîne au niveau général de la compréhension de l'univers créé. Nous reviendrons, en revanche, sur la structure symbolique de la lettre qui en arrive, peu à peu, par cette suite de jeux formels, à fonder toute une tradition d'interprétation visuelle projetée au premier plan par la succession des figures colorées sur la peau du parchemin. Dans ses Étymologies (29, 1-3), Isidore de Séville notait : « ... l'étymologie est l'origine des vocables quand on saisit la force signifiante d'un verbe ou d'un nom (vis verbi vel nominis) au moyen de sa dérivation (interpretatio) ». Le sens, c'est-à-dire le contenu, est associé à vis, l'énergie, la force, la puissance dynamique, la substance des êtres et des choses. Cette substance doit être retrouvée dans leur nom même (I, 7) ${ }^{9}$. Par la similitude des signes graphiques, il faut savoir remonter jusqu'à leur origine naturelle, dont la lettre seule peut être le principe tout à la fois dynamique et régénérateur. Le privilège ainsi accordé à la lettre, et aussi au geste d'écrire, par Isidore de Séville demeure tout au long de son encyclopédie : la lettre a une telle puissance, en tant que signe, qu'elle nous transmet la parole de l'absent sans le secours de la voix ${ }^{10}$. 
7 Sur les feuillets 2 recto et 16 recto du manuscrit 1 , le « V » initial renvoie à vis, puis à vir, à Jean de Réome, l'homme de Dieu, puis à Dieu lui-même. Le mot vir est substitué à la parole du premier, que son hagiographe dit être " en train de prêcher ", praedicandus, puis à la parole de Dieu. L'initiale en vient à contenir, puis à libérer, toute cette force retenue dans le mot et, au-delà, dans le texte consigné par écrit. Les formes enroulées autour des corps des lettres, les taches de couleurs, et les lettres animales, tout participe du même effort permanent de créativité, sur les bords du texte réglé, parfois aussi contre le texte, quand il y a empiètement sur le signe écrit, gêne pour sa lecture, remise en question des principes de composition du feuillet. Au feuillet 11 verso, un petit dragon à queue enroulée devient le «V » initial de Vir quidam nobili intentione praeventus sequanus nomine... La lettre animale, démoniaque ou monstrueuse, est génératrice de formes quasiment à l'infini.

8 Ce sont là deux aspects d'une question plus large que nous tenions, toutefois, à souligner. Nous les rattacherons aux pratiques de l'étymologie comme mode de lecture et d'interprétation plus générale ${ }^{11}$. Le Verbe divin se révèle à travers une profusion de formes et de signes, celles-là vues en toute indépendance de celles-ci, créant un riche écrin visuel à une manifestation de puissance créatrice, en écho lointain au geste initial et au souffle de l'esprit. Sur le feuillet 16 recto, les tiges sont enroulées autour des deux branches de la lettre «V »; la hampe du «I » s'achève par le bec d'un oiseau ou d'un autre animal, qui mord le bas de la tige; une forme de dragon se saisit de la partie haute. Même indépendantes de la lettre, ces formes, ces figures sont contraintes par elle, fixées à leur juste place et situées au mieux: sur un cadre; dans un espace nettement délimité.

9 La question est désormais posée : la contrainte vient-elle de l'extérieur ? S'agirait-il vraiment d'un "art dirigé »? Ou est-elle pensée par le moine, induite de l'intérieur même du livre manuscrit par des pratiques monastiques liées aux jeux des formes et des figures ${ }^{12}$, en prise directe aussi avec les usages du scriptorium? 


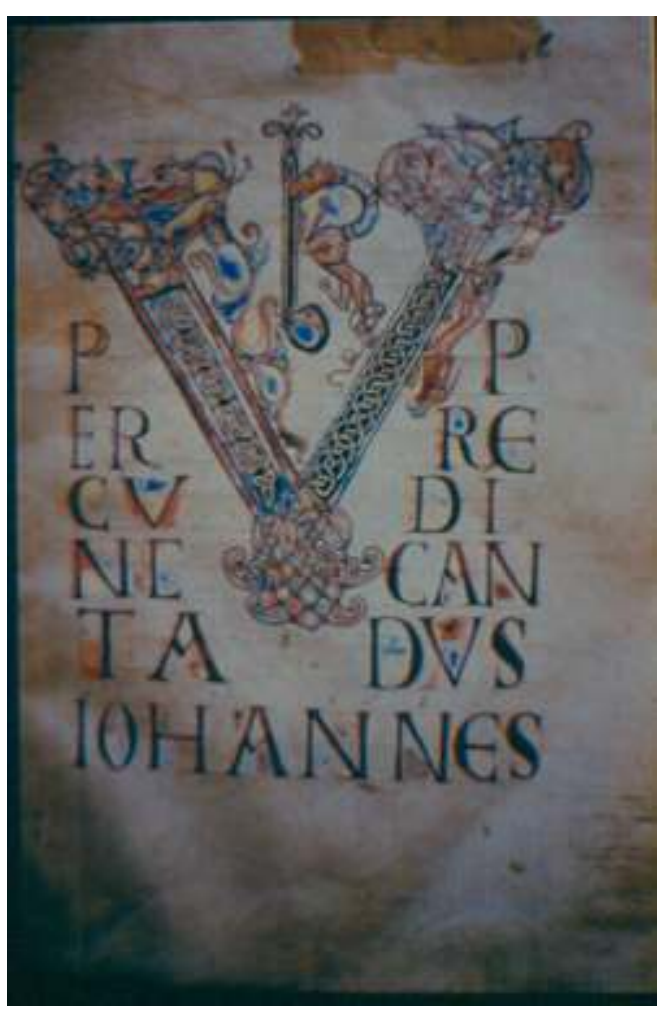

Vie de saint Jean de Réome, Mélanges sur l'histoire de Moutiers-Saint-Jean, (Début du $x^{e}$ siècle ; Semur, Bibliothèque municipale, manuscrit 1, fol. 16 r.)

\section{NOTES DE FIN}

1. Quatre manuscrits doivent, en particulier, retenir l'attention : le manuscrit 1 , Vie de saint Jean de Réome et Mélanges sur l'histoire de Moutiers-Saint-Jean (fin $\mathrm{x}^{\mathrm{e}}$-déb. $\mathrm{XI}^{\mathrm{e}}$ siècle) ; le manuscrit 24, le Martyrologe de Moutiers-Saint-Jean (XII ${ }^{\mathrm{e}}$ siècle) ; le manuscrit 34, un missel du XIV ${ }^{\mathrm{e}}$ siècle ; le manuscrit 97, les Psaumes de la Pénitence décorés d'une danse macabre (XVI ${ }^{\mathrm{e}}$ siècle). I. LOUVET, "Semur-en-Auxois, Bibliothèque municipale ", in Patrimoine des Bibliothèques de France, vol. 5 : Auvergne, Bourgogne, Rhônes-Alpes, Paris, Payot, 1995, p. 206-209. Pour la Vita de saint Jean de Réome, JONAS DE BOBBIO, Vita Joannis abbatis, in M.G.H., Scriptorum rerum merovingicarum, III, p. 502-517.

2. P. STIRNEMANN, « L'illustration du commentaire d'Haymon sur Ezéchiel. Paris, B. n. latin 12302 », in L'école carolingienne d'Auxerre de Murethach à Rémi, 830-908, Paris, Beauchesne, 1991, p. 93-117 (Entretiens d'Auxerre, 1989).

3. Entre autres exemples, les manuscrits 7 à 9 des Moralia in Job de saint Grégoire le Grand à la Bibliothèque municipale de Chalon-sur-Saône, et le manuscrit 2, dit de la Bible de Saint-Bénigne de Dijon à la Bibliothèque municipale de Dijon. M. PORTELLI, Étude sur les manuscrits 7 à 9 de la Bibliothèque municipale de Semur-en-Auxois, provenant de l'abbaye de La Ferté-sur-Grosne, mémoire de Maîtrise, Université de Bourgogne, Dijon, 
1994 (F. Joubert, dir.) ; Y. ZALUSKA, L'enluminure et le scriptorium de Cîteaux au XII siècle, in Commentarii cistercienses, 1989.

4. Sur les notions de sens et de contrôle exercé par l'Église, H. TOUBERT, Un art dirigé. Réforme grégorienne et iconographie, Paris, éd. du Cerf, 1990 (trad. it., Milan, éd. Jaca Book, 2001) ; W. CAHN, Studies in Medieval Art and Interpretation, Londres, Pindar Press, 2000, en part. " The rule and the Book. Cistercian Book Illumination in Burgundy and Champagne ", " Architecture and Exegesis. Richard of Saint-Victor's Ezekiel Commentary an its Illustrations », p. 204-238, p. 369-406. Sur l'importance de la question formelle pour objectiver le manuscrit et lui conférer un statut propre, E. VINAVER, À la recherche d'une poétique médiévale, Paris, Nizet, 1970, puis les travaux qui en ont découlé.

5. Histoire de l'abbaye de Lérins, M. LABROUSSE, E. MAGNANI, Y. CODOU, J.-M. LE GALL, R. BERTRAND, Dom V. GAUDRAT, Abbaye de Bellefontaine-ARCCIS, 2005, p. 21-124 (Cahiers cisterciens. Des lieux et des temps, 9).

6. Dans la fabrication même du feuillet de manuscrit, tout est matière : la peau du veau, le vélin ; les pigments des couleurs à base de minéraux ou de végétaux, le blanc provenant de la céruse de plomb, la sanguine ou rouge de la laque obtenue du jus de lierre cuit, le bleu du jus de bleuet et de la guède.

7. J. FONTAINE, Isidore de Séville et la culture classique dans l'Espagne wisigothique, Paris, Institut des Études augustiniennes, 3 vol., 1959-1983, vol. 1, p. 57-58, pour ces usages jusqu'à Isidore.

8. E. VINAVER, À la recherche d'une poétique médiévale, op. cit.; J. BALTRUSAITIS, Réveils et prodiges dans l'art médiéval, Paris, A. Colin, 1960, chap. 6, p. 195-235 (« Le réveil du fantastique dans le décor du livre »).

9. De nomine : Nisi enim nomen scieris, cognitio rerum perit.

10. Sur ces contaminations voir Ed. JABÈs, El, ou le Dernier Livre, Paris, Gallimard, 1973, p. 44, à propos du pronom féminin el ou ele et le substantif homonyme ele ('aile') : El/ Ailes brisées.

11. Nous renverrons aux travaux de J. PÉPIN, entre autres à son article, "L'herméneutique ancienne », in Poétique 23, 1975, p. 291-300. Pour une position plus globale de la question, H. INGLEBERT, Interpretatio Christiana. Les mutations des savoirs (cosmographie, géographie, ethnographie, histoire) dans l'Antiquité chrétienne, 30-360 ap. J.-C., Paris, Institut des Études augustiniennes, 2001.

12. Jeux de mémoire. Aspects de la mnémotechnie médiévale, B. ROY, P. ZUMTHOR (dir.), Montréal, Vrin/Les Presses de l'Université de Montréal, 1985 ; M. CARRUTHERS, The Book of Memory. A Study of Memory in Medieval Culture, Cambridge University Press, 1990 (trad. fr., D. Meur, Paris, éd. Macula, 2002).

\section{INDEX}

Mots-clés : Jean de Réome (saint), vie de saint Jean de Réome Index géographique : France/Moutiers-Saint-Jean 